\title{
FUSE spectroscopy of the sdOB primary of the post common-envelope binary LB 3459 (AA Doradus) ${ }^{\star}$
}

\author{
J. Fleig ${ }^{1}$, T. Rauch ${ }^{1}$, K. Werner ${ }^{1}$, and J. W. Kruk ${ }^{2}$ \\ ${ }^{1}$ Institute for Astronomy and Astrophysics, Kepler Center for Astro and Particle Physics, Eberhard Karls University, Sand 1, \\ 72076 Tübingen, Germany \\ e-mail: rauch@astro.uni-tuebingen.de \\ 2 Department of Physics and Astronomy, Johns Hopkins University, Baltimore, MD 21218, USA
}

Received 2 August 2008 / Accepted 5 September 2008

\section{ABSTRACT}

\begin{abstract}
Context. LB 3459 (AA Doradus) is an eclipsing, close, post common-envelope binary (PCEB) consisting of an sdOB primary star and an unseen secondary with an extraordinarily low mass $\left(M_{2} \approx 0.066 M_{\odot}\right)$ - formally a brown dwarf. A recent NLTE spectral analysis shows a discrepancy with the surface gravity, which is derived from analyses of radial-velocity and lightcurves.

Aims. We aim at precisely determining of the photospheric parameters of the primary, especially of the surface gravity, and searching for weak metal lines in the far UV.

Methods. We performed a detailed spectral analysis of the far-UV spectrum of LB 3459 obtained with FUSE by means of state-ofthe-art NLTE model-atmosphere techniques.

Results. A strong contamination of the far-UV spectrum of LB 3459 by interstellar line absorption hampers a precise determination of the photospheric properties of its primary star. Its effective temperature $\left(T_{\text {eff }}=42 \mathrm{kK}\right)$ was confirmed by the evaluation of new ionization equilibria. For the first time, phosphorus and sulfur have been identified in the spectrum of LB 3459. Their photospheric abundances are solar and 0.01 times solar, respectively. From the C III $\lambda \lambda 1174-1177 \AA$ multiplet, we can measure the rotational velocity $v_{\mathrm{rot}}=35 \pm 5 \mathrm{~km} \mathrm{~s}^{-1}$ of the primary of LB 3459 and confirm that the rotation is bound. From a re-analysis of optical and UV spectra (analogue to Rauch 2000, A\&A, 356, 665), we determine a slightly higher surface gravity $\log g=5.3 \pm 0.1$ compared to Rauch $(2000, \log g=5.2 \pm 0.1)$.

Conclusions. The rotational velocity of the primary of LB 3459 is consistent with a bound rotation. The higher log $g$ reduces the discrepancy in mass determination in comparison to analyses of radial-velocity and lightcurves. However, the problem is not completely solved.
\end{abstract}

Key words. stars: abundances - stars: atmospheres - stars: binaries: eclipsing - stars: early-type - stars: low-mass, brown dwarfs stars: individual: LB 3459 (AA Doradus)

\section{Introduction}

The eclipsing binary system LB 3459 (AA Doradus) is a blue foreground object of the LMC at a spectroscopic distance of $d=396$ pc (Rauch 2000). It consists of aa sdOB with an effective temperature of $T_{\text {eff }}=42 \mathrm{kK}$ and a low-mass companion from which no direct spectroscopic information has been obtained yet. Due to its mass of about $0.066 M_{\odot}$ it formally lies in the browndwarf range. The orbital period is about $0.26 \mathrm{~d}$ and the inclination is $i=90^{\circ}$.

The system was analyzed several times, starting with Kilkenny et al. (1979, 1981) and Paczynski (1980), who established the basic parameters. First NLTE analyses of the primary were performed by Kudritzki et al. (1982) and Lynas-Gray et al. (1984). Details on the history of this object can be found in Rauch $(2000,2004)$.

Recent spectral analyses by means of NLTE modelatmospheres that were based on optical (ESO CASPEC $\left.{ }^{1}\right)$ and ultraviolet ( $\mathrm{IUE}^{2}$ ) observations (Rauch 2000) have shown a

* Based on observations made with the NASA-CNES-CSA Far Ultraviolet Spectroscopic Explorer. FUSE is operated for NASA by the Johns Hopkins University under NASA contract NAS5-32985.

${ }^{1}$ European Southern Observatory, Cassegrain Echelle Spectrograph.

2 International Ultraviolet Explorer. discrepancy with analyses of radial-velocity and lightcurves (Hilditch et al. 1996, 2003). Rauch (2000) determined a surface gravity of $\log g=5.21 \pm 0.1$, while Hilditch et al. (2003) obtained $\log g=5.45-5.51$ from lightcurve and mass function. Because the analysis of Rauch (2000) suffered from the long exposure times (some hours) hence smearing, due to the orbital movement of the available spectra, Rauch \& Werner (2003) measured the radial-velocity curve from optical spectra with short (180 s) exposure times obtained with ESO's VLT ${ }^{3}$ and UVES ${ }^{4}$. They obtained a radius $r_{1}=0.169 R_{\odot}$ which is smaller than the $r_{1}=0.236 R_{\odot}$ found by Rauch (2000). Since the stellar radius depends on $g$ this may be a hint of a higher value than $\log g=5.21$. However, data reduction of the Balmer lines in the UVES spectra used by Rauch \& Werner (2003) was not very accurate, so a precise determination of $\log g$ of the primary by means of NLTE modeling techniques is still lacking.

Consequently, high-resolution and high-S/N observations (exposure times of $200 \mathrm{~s}$ each) in the far-UV range were performed with the FUSE ${ }^{5}$ satellite. The FUSE wavelength range $(904 \AA<\lambda<1187 \AA$ ) covers the hydrogen Lyman series

\footnotetext{
3 Very Large Telescope.

${ }^{4}$ Ultraviolet and Visual Echelle Spectrograph.

5 Far Ultraviolet Spectroscopic Explorer.
} 
except for $\operatorname{Ly} \alpha$. The series decrement is a sensitive indicator for $\log g$.

A detailed spectral analysis of the FUSE observation of LB 3459 is described in (Sect. 4). Since the far-UV spectrum of LB 3459 turned out to be strongly contaminated by interstellar absorption, we modeled the ISM line absorption (Sect. 2) in order to distinguish weak lines of iron-group elements (here: $\mathrm{Ca}$, $\mathrm{Sc}, \mathrm{Ti}, \mathrm{V}, \mathrm{Cr}, \mathrm{Mn}, \mathrm{Fe}, \mathrm{Co}, \mathrm{Ni}$; Sect. 4).

\section{The far-UV spectrum of LB 3459}

The FUSE instrument consists of four independent, co-aligned telescopes and spectrographs. Taken together, the four channels span the wavelength range $904 \AA<\lambda<1187 \AA$ with a typical resolving power of $R \approx 20000$. Further information on the FUSE mission and instrument can be found in Moos et al. (2000) and Sahnow et al. (2000).

Far-UV observations were performed with FUSE on August 29, 2003 (observation id: D0250101) and June 22, 2004 (id: D0250102) using the LWRS aperture with a resolving power of $R \approx 20000$. The individual exposure times were about $200 \mathrm{~s}$ to minimize effects of orbital motion. Problems with coalignment of the telescope channels in the second observation resulted in loss of the $\mathrm{SiC}$ channel data and most of the LiF2 channel data; the total exposure times varied from $730 \mathrm{~s}$ in the SiC channels to $2335 \mathrm{~s}$ in LiF1. The data were reduced with CalFUSE v. 3.1.3, but a subsequent reduction with the final version of CalFUSE, v. 3.2.2, did not result in any significant changes to the spectrum. A correction for the "worm" feature (a shadow cast by the detector grid wires) in LiF1b was obtained from a highly-smoothed ratio of the LiF2a to LiF1b spectra. For a representative discussion of FUSE data reduction procedures, see Kruk et al. (2002), and Dixon et al. (2007) for a description of CalFUSE.

An additional far-UV observation was performed earlier with $\mathrm{BEFS}^{6}(R \approx 10000)$ aboard ORFEUS II ${ }^{7}$ on November 30, 1996 (id: BEFS2162) with an exposure time of $1112 \mathrm{~s}$. We retrieved it from MAST ${ }^{8}$. In Fig. 1 we show a comparison of ORFEUS and FUSE observations. We note that the measured flux levels agree very well. For our analysis we mainly used the FUSE observation that has better resolution and S/N. All observations are slightly smoothed with a Savitzky-Golay low-pass filter (Savitzky \& Golay 1964).

The FUSE spectrum exhibits a strong contamination by interstellar line absorption (Fig. 2). To identify weak photospheric lines in the spectrum, we employed the program OWENS (cf. Lemoine et al. 2002; Hébrard et al. 2002). With OWENS, we can simulate interstellar clouds with individual parameters such as, e.g., radial velocity, column density in the line of sight, temperature of the gas, and microturbulence velocity. A large number of ions were taken into account, e.g. $\mathrm{H}$ I, C II, C III, N I, N II, N III, $\mathrm{O}$ I, Si II, Ar I, Fe II, and the $\mathrm{H}_{2}$ molecule $(J=0,1,2,3,4)$. The continuum is well matched and most of the absorption lines are well reproduced by the combined spectrum (Fig. 2). The stellar spectrum is calculated with Kurucz' LIN lines (cf. Sect. 4) and, thus, the strong absorption feature at $979 \AA$ (not observed) is most likely due to uncertain wavelengths in these lists.

Rauch (2000) determined an interstellar neutral hydrogen column density of $n_{\mathrm{HI}}=2 \times 10^{20} \mathrm{~cm}^{-2}$ from Ly $\alpha$ (IUE observation). We measured the same value from Ly $\beta$ in the FUSE

\footnotetext{
${ }^{6}$ Berkeley Extreme and Far-UV Spectrometer.

7 Orbiting and Retrievable Far and Extreme Ultraviolet Spectrometer.

${ }^{8}$ Multimission Archive at the Space Telescope Science Institute.
}

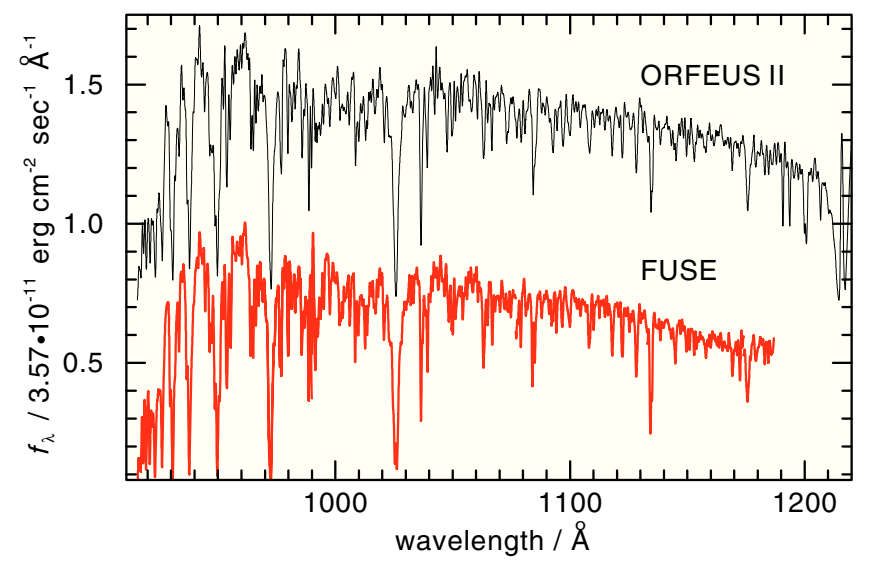

Fig. 1. Comparison of ORFEUS (shifted by 0.7 to the top) and FUSE observations of LB 3459 .

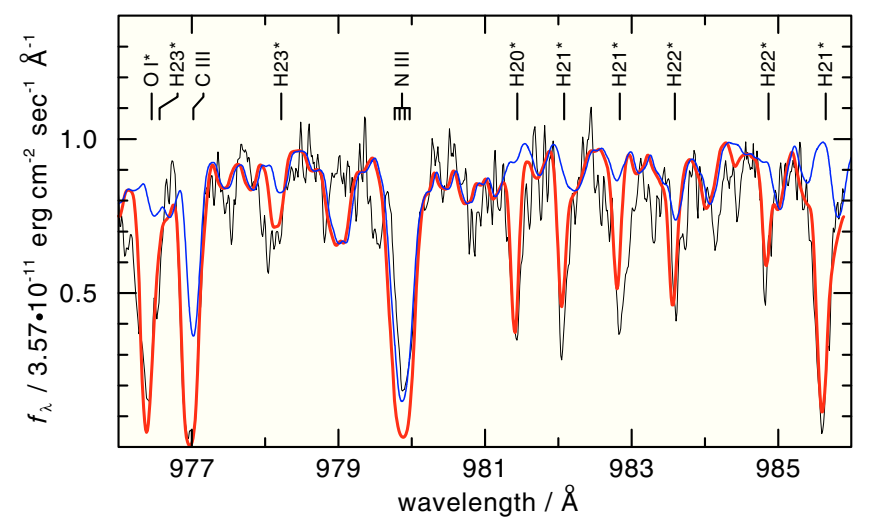

Fig. 2. Comparison of the synthetic stellar spectrum (thin line, blue) and the combined synthetic stellar + ISM spectrum (thick, red) with a section of the FUSE observation of LB 3459. Interstellar lines are labeled with an asterisk.

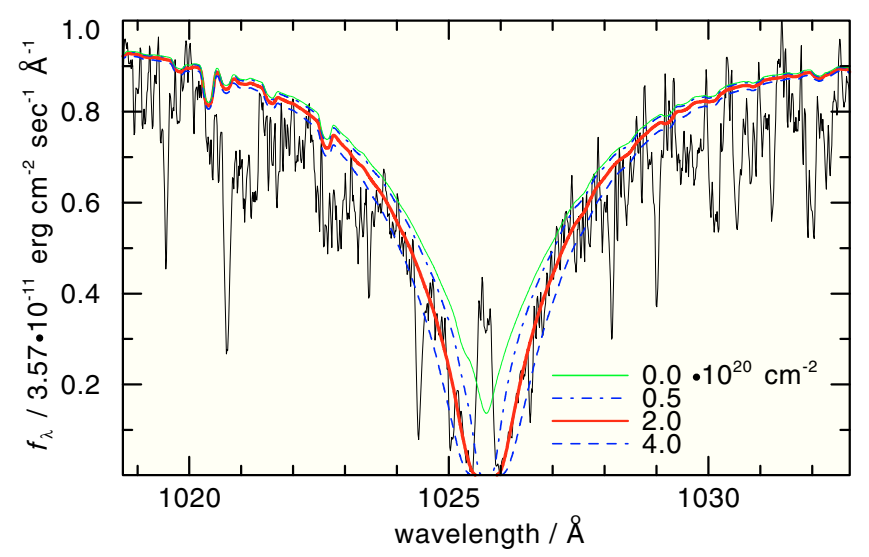

Fig. 3. Comparison of our synthetic Ly $\beta$ line profile $\left(T_{\text {eff }}=42 \mathrm{kK}\right.$, $\log g=5.3$ ) at different $n_{\mathrm{HI}}$ with the FUSE observation. Note that the inner line core appears much too broad at $n_{\mathrm{HI}}=4 \times 10^{20} \mathrm{~cm}^{-2}$.

observation (Fig. 3). The inner line core appears much too broad at $n_{\mathrm{HI}}=4 \times 10^{20} \mathrm{~cm}^{-2}$.

In order to determine the interstellar reddening, we used FUSE and IUE observations (Fig. 4). We find that the continuum slope is best reproduced with $E_{B-V}=0.01 \pm 0.01$ using the Galactic reddening law of Seaton (1979). At this low $E_{B-V}$, we arrive at the same result if the LMC reddening law of Howarth (1983) is used. This value is significantly lower than 


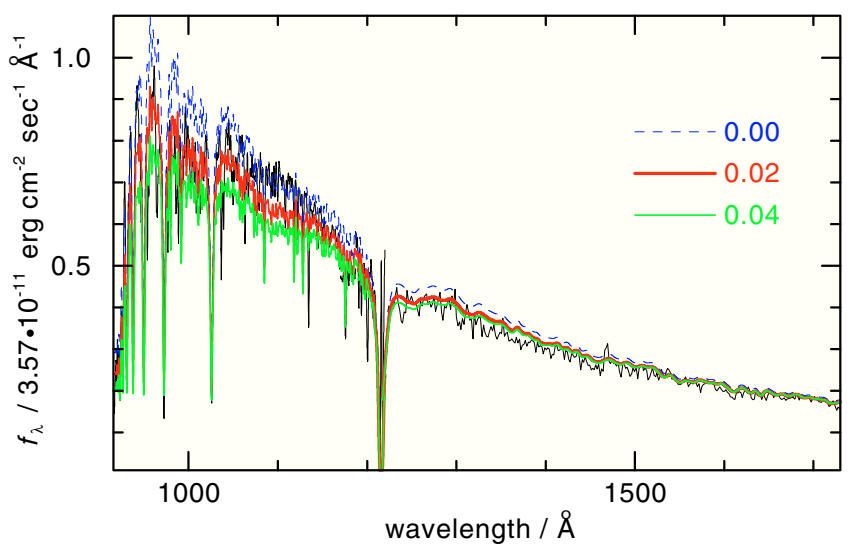

Fig. 4. Comparison of our synthetic spectrum calculated with Kurucz' LIN lines (cf. Sect. 4$)$ with the FUSE $(\lambda<1187 \AA)$, ORFEUS, and IUE $(\lambda>1220 \AA$, SWP04887LL retrieved from INES) observations at three different $E_{B-V}$. The IUE observation is scaled by a factor of 1.06 to match flux calibrations of other UV satellites (see, e.g., Kruk et al. 1997). The synthetic spectra are normalized to match the flux level of the IUE observation at $\lambda=1700 \AA$. For clarity, at $\lambda<1220 \AA$ the synthetic spectra and the observation are convolved with a Gaussian of $1 \AA(F W H M)$. At $\lambda>1220 \AA$ the synthetic spectra are convolved with a Gaussian of $7 \AA(F W H M)$ to simulate IUE's low resolution.

$E_{B-V}=0.0526$ (cf. Rauch 2000), calculated from $n_{\mathrm{HI}}$ using an approximate formula given by Groenewegen \& Lamers (1989).

The ISM line absorption has been neglected in this particular determination. Its consideration would decrease the flux level of our synthetic energy distribution at higher energies and, thus, the derived reddening would be even lower. Since there are uncertainties, such as in the location of the observed stellar continuum-flux level (difficult to determine in the presence of strong ISM absorption) and the validity of the applied reddening law, and the reddening is relatively small, we decided to adopt $E_{B-V}=0.00$ for our analysis without loss of generality. Our determination of photospheric properties is not significantly affected by this assumption.

Figure 7 shows that - even considering ISM line absorptionan additional, presently still unexplained factor, linearly increasing towards longer wavelengths, appears necessary to achieve a good fit to the continuum in the FUSE wavelength range.

\section{Atomic data and modeling}

The model atmosphere of the primary of LB 3459 was calculated with the Tübingen Model Atmosphere Package TMAP. Details about TMAP are given by Werner et al. (2003) and Rauch \& Deetjen (2003).

In addition to $\mathrm{H}$ and $\mathrm{He}$, the light metals $\mathrm{C}, \mathrm{N}, \mathrm{O}, \mathrm{Mg}, \mathrm{Si}$, $\mathrm{P}$, and $\mathrm{S}$, as well as the elements of the iron group $(\mathrm{Ca}-\mathrm{Ni})$, were considered. In our final model atmosphere, 567 levels are treated in NLTE with 724 individual lines and 801 superlines. The statistics of our final model atmosphere are summarized in Table 1. The model atoms are based on atomic data taken from the databases of NIST ${ }^{9}$ (National Institute of Standards and Technology), Opacity Project ${ }^{10}$ (Seaton et al. 1994), and Iron Project $^{11}$ (Hummer et al. 1993). In the case of the iron-group

\footnotetext{
9 http://physics.nist.gov

10 http://vizier.u-strasbg.fr/topbase/topbase.html

11 http://vizier.u-strasbg.fr/tipbase/tipbase.html
}

Table 1. Statistics of the elements considered in our final model.

\begin{tabular}{|c|c|c|c|c|c|c|c|}
\hline Ion & $\overline{\mathrm{NL}}$ & $\overline{\mathrm{L}}$ & $\overline{\mathrm{RBB}}$ & Ion & $\overline{\mathrm{NL}}$ & $\overline{\overline{\mathrm{RBB}}}$ & $\overline{\mathrm{RBB}_{\mathrm{st}}}$ \\
\hline$\overline{\mathrm{H} \mathrm{I}}$ & 15 & 1 & 105 & Ca II & 7 & 21 & 890 \\
\hline H II & 1 & 0 & 0 & Ca III & 7 & 25 & 10241 \\
\hline $\mathrm{He} \mathrm{I}$ & 29 & 15 & 61 & $\mathrm{Ca}$ IV & 7 & 22 & 20291 \\
\hline He II & 14 & 18 & 91 & $\mathrm{Ca} \mathrm{V}$ & 7 & 24 & 141956 \\
\hline He III & 1 & 0 & 0 & $\mathrm{Ca}$ VI & 1 & 0 & 0 \\
\hline $\mathrm{C}$ II & 1 & 41 & 0 & Sc II & 7 & 26 & 36087 \\
\hline $\mathrm{C}_{\text {III }}$ & 13 & 54 & 19 & Sc III & 7 & 20 & 675 \\
\hline C IV & 14 & 44 & 35 & Sc IV & 7 & 26 & 15024 \\
\hline $\mathrm{C} \mathrm{V}$ & 1 & 0 & 0 & $\mathrm{Sc} \mathrm{V}$ & 7 & 24 & 65994 \\
\hline $\mathrm{N}_{\text {II }}$ & 1 & 21 & 0 & Sc VI & 1 & 0 & 0 \\
\hline N III & 13 & 32 & 21 & Ti II & 7 & 26 & 154681 \\
\hline $\mathrm{N}$ IV & 34 & 60 & 108 & Ti III & 7 & 24 & 21722 \\
\hline $\mathrm{N} \mathrm{V}$ & 14 & 48 & 35 & Ti IV & 7 & 24 & 1000 \\
\hline $\mathrm{N}$ VI & 1 & 0 & 0 & $\mathrm{TiV}$ & 7 & 24 & 26654 \\
\hline O II & 2 & 3 & 0 & Ti VI & 1 & 0 & 0 \\
\hline O III & 19 & 35 & 33 & V IV & 7 & 24 & 37130 \\
\hline O IV & 11 & 58 & 19 & $\mathrm{~V} \mathrm{~V}$ & 7 & 27 & 2123 \\
\hline $\mathrm{OV}$ & 5 & 7 & 3 & V VI & 1 & 0 & 0 \\
\hline $\mathrm{O}$ VI & 1 & 0 & 0 & $\mathrm{Cr}$ II & 6 & 18 & 303129 \\
\hline $\mathrm{Mg}_{\mathrm{I}}$ & 5 & 1 & 3 & $\mathrm{Cr}$ III & 7 & 25 & 523586 \\
\hline Mg II & 14 & 19 & 34 & Cr IV & 7 & 25 & 234170 \\
\hline Mg III & 15 & 20 & 20 & $\mathrm{Cr} \mathrm{V}$ & 7 & 24 & 43860 \\
\hline Mg IV & 1 & 0 & 0 & Cr vI & 1 & 0 & 0 \\
\hline Si III & 6 & 6 & 4 & Mn II & 6 & 20 & 68101 \\
\hline Si IV & 16 & 7 & 44 & Mn III & 7 & 22 & 671822 \\
\hline $\mathrm{Si} \mathrm{V}$ & 1 & 0 & 0 & Mn IV & 7 & 25 & 719387 \\
\hline $\mathrm{P}_{\text {III }}$ & 3 & 7 & 0 & Mn V & 7 & 25 & 285376 \\
\hline P IV & 15 & 36 & 9 & Mn VI & 1 & 0 & 0 \\
\hline $\mathrm{P} \mathrm{V}$ & 18 & 7 & 12 & $\mathrm{Fe}$ II & 6 & 20 & 218490 \\
\hline P VI & 1 & 0 & 0 & Fe III & 7 & 25 & 301981 \\
\hline S III & 1 & 9 & 0 & $\mathrm{Fe}$ IV & 7 & 25 & 1027793 \\
\hline S IV & 6 & 9 & 4 & $\mathrm{Fe} \mathrm{V}$ & 7 & 25 & 793718 \\
\hline $\mathrm{S}$ V & 14 & 5 & 16 & Fe VI & 1 & 0 & 0 \\
\hline S VI & 18 & 7 & 48 & Co II & 6 & 18 & 244873 \\
\hline \multirow[t]{9}{*}{ S VII } & 1 & 0 & 0 & Co III & 7 & 25 & 679280 \\
\hline & & & & Co IV & 7 & 25 & 552916 \\
\hline & & & & Co v & 7 & 25 & 1469717 \\
\hline & & & & Co VI & 1 & 0 & 0 \\
\hline & & & & Ni II & 6 & 18 & 96647 \\
\hline & & & & Ni III & 7 & 22 & 418248 \\
\hline & & & & Ni IV & 7 & 25 & 949506 \\
\hline & & & & $\mathrm{Ni} \mathrm{V}$ & 7 & 27 & 1006189 \\
\hline & & & & $\mathrm{Ni}$ VI & 1 & 0 & 0 \\
\hline
\end{tabular}

For iron group elements, $\mathrm{RBB}_{\text {st }}$ denotes the number of individual lines which were summed up to so-called superlines (see, e.g., Rauch \& Deetjen 2003).

elements, energy levels and oscillator strengths stem from Kurucz' line lists (Kurucz 1991, see Sect. 4 for details).

We adopted the parameters determined by Rauch (2000) ( $T_{\text {eff }}=42 \mathrm{kK}, \log g=5.21$, and abundances $)$ for our first models. For additional species (Ca, Sc, Ti, V, Cr, Mn, Co), we assumed solar abundances (Asplund et al. 2005). Figure 5 shows the ionization fractions of all elements in the model atmosphere. The ionization stages III and IV dominate in the line-forming regions $(\log m \approx-1)$ for the light metals, whereas IV and $\mathrm{V}$ are most populated in the case of the iron-group elements.

A first test calculation was performed to check whether we can reproduce the results of Rauch (2000) with our more elaborated models (e.g. we consider for $\mathrm{Fe}$ and $\mathrm{Ni}$ opacities of the ionization stages II-VI in contrast to IV-IX). Figure 6 demonstrates that the IUE near-UV observation is reproduced well with adjusted $\mathrm{Si}$ and $\mathrm{Ni}$ abundances (about a factor of two lower and a factor of two higher, respectively, compared to the previous results). 


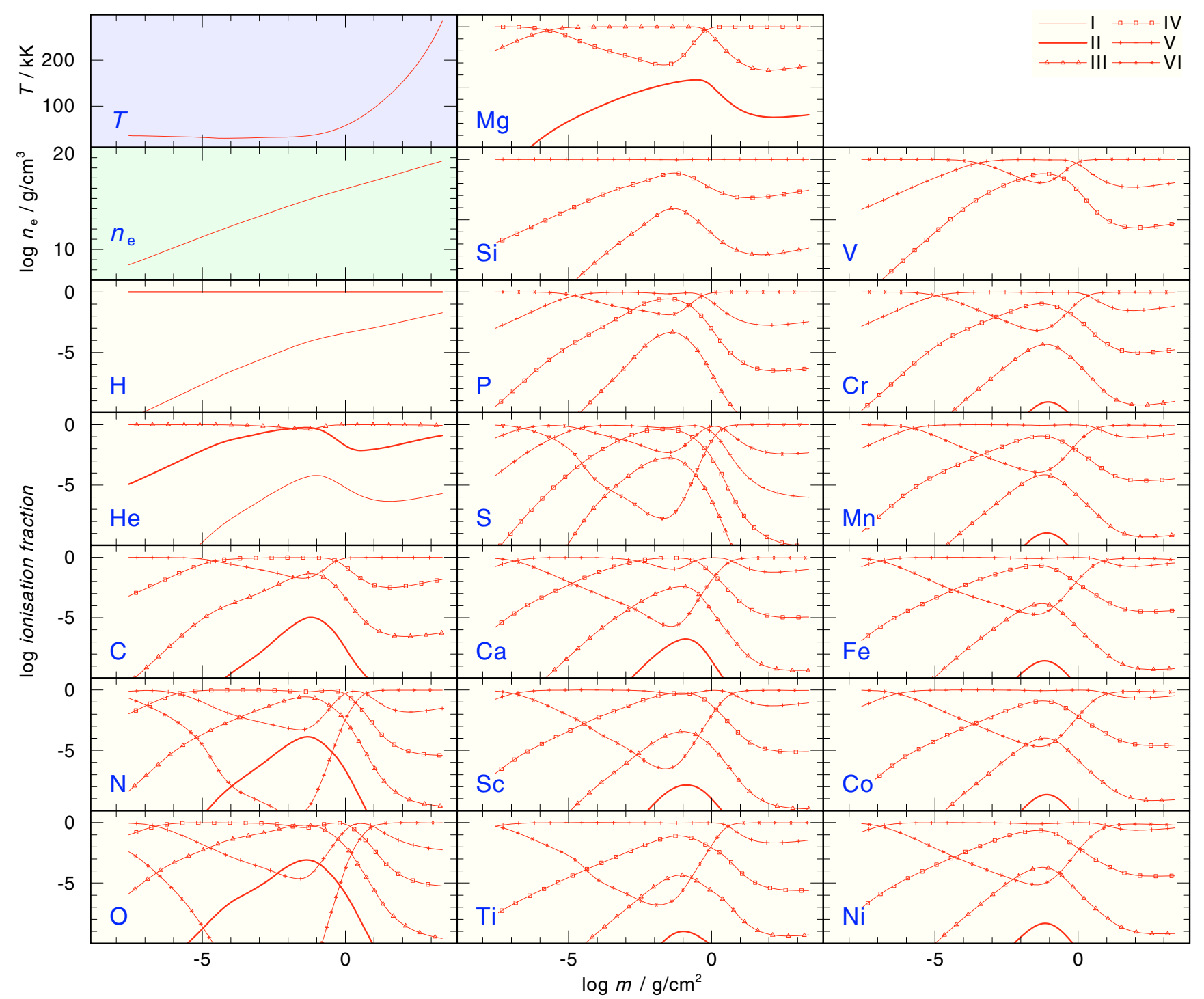

Fig. 5. Temperature and density structure, as well as ionization fractions of the elements that are considered in our atmosphere model of LB 3459.

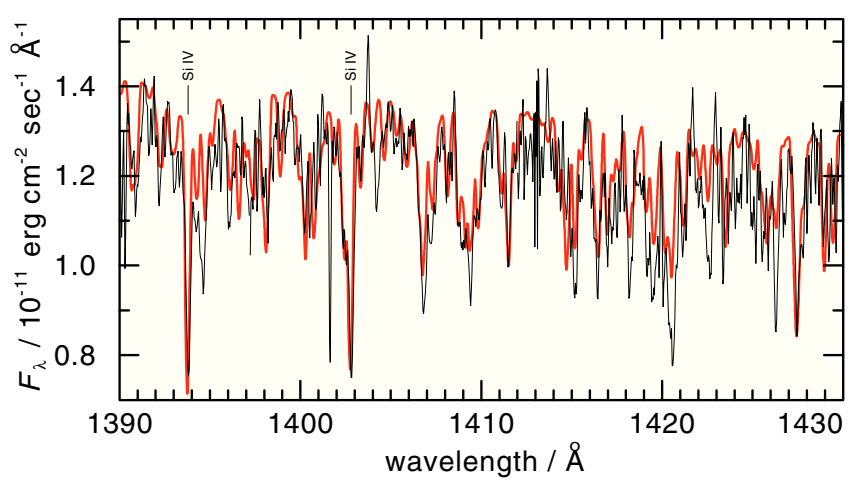

Fig. 6. Comparison of our synthetic spectrum (thick, red line, $T_{\text {eff }}=42 \mathrm{kK}, \log g=5.3$, abundances see Fig. 14) with the IUE observation (thin line, SWP 17822, cf. Rauch 2000, his Fig. 14) of LB 3459.

For a combined synthetic spectrum that includes both the synthetic stellar as well as the ISM spectrum, we first normalized the ISM spectrum calculated by OWENS and multiplied it by the TMAP spectrum, which has been convolved with a rotational profile $\left(35 \mathrm{~km} \mathrm{~s}^{-1}\right.$ unless otherwise noted, cf. Sect. 4.1) before. Finally, the combined spectrum was convolved with a Gaussian of $0.05 \AA(F W H M)$ to match the instrument's resolution.
The apparent continuum slope in the FUSE spectrum changes at approximately $1100 \AA$, becoming noticeably shallower on the shorter-wavelength side of this point. To facilitate detailed comparison of the small-scale structure in the models with the data, the models shown in Fig. 7 have been normalized by a factor that increases linearly from $5.6 \times 10^{18}$ at $910 \AA$ to $7.9 \times 10^{18}$ at $1190 \AA$.

\subsection{Identification of photospheric lines}

In the FUSE observation of LB 3459 (Fig. 7), several photospheric absorption lines are prominent and isolated from ISM absorption lines. In Table 2, we summarize the identified photospheric lines. For the first time, we have identified $\mathrm{P}_{\text {IV }}$ and $\mathrm{P} \mathrm{V}$ (Fig. 8), as well as S IV and S vi (Fig. 9), lines in the spectrum of LB 3459 .

Rauch (2000) identified a large number of strong absorption lines of Fe IV-V and Ni IV-V in IUE observations of LB 3459. In the FUSE observation we cannot identify isolated lines of the iron-group elements because only weak lines are located in this wavelength range, and the strong ISM line absorption hampers the search for such weak lines. 


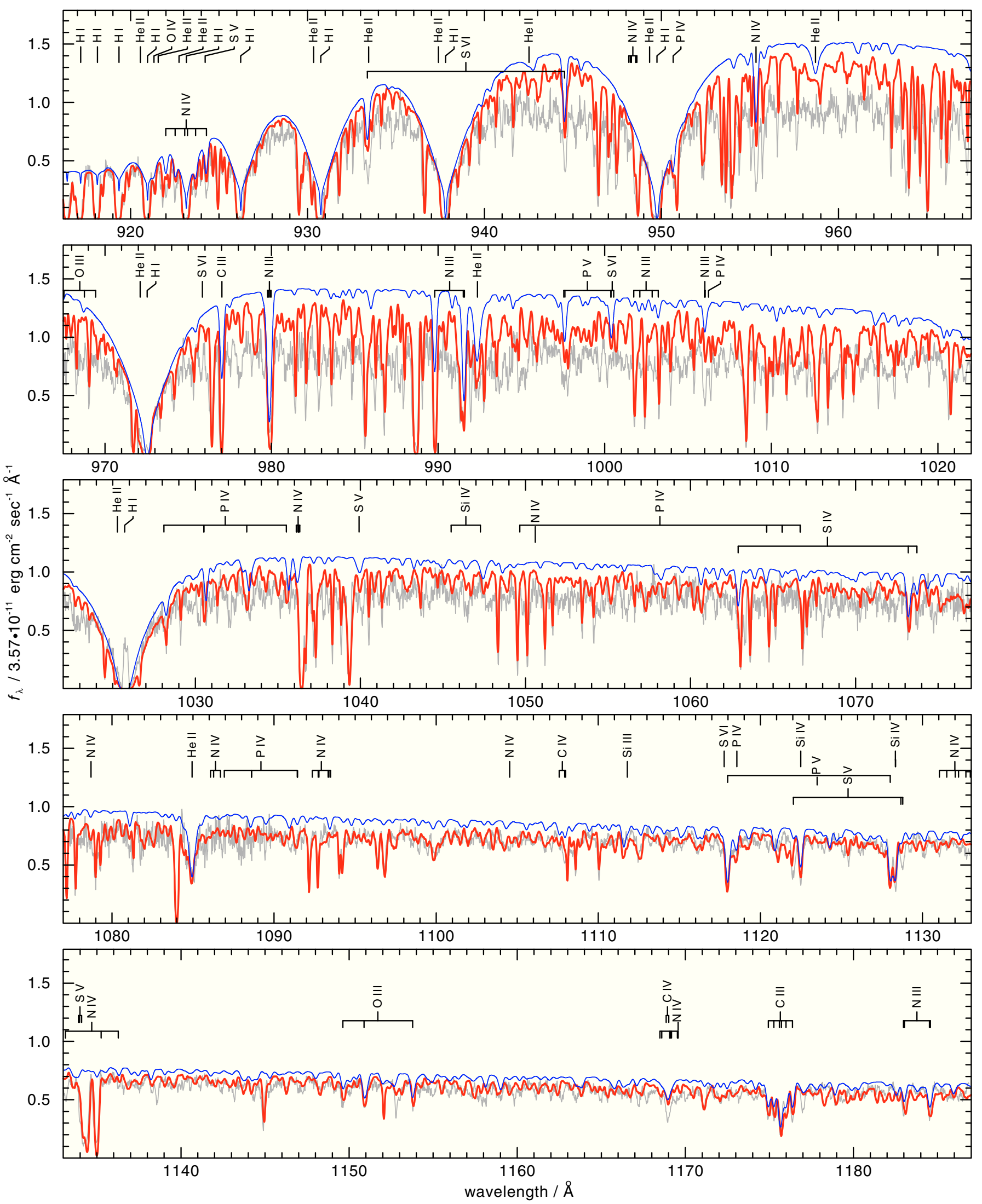

Fig. 7. Comparison of the synthetic stellar spectrum of our final HHeCNOMgSiPS + CaScTiVCrMnFeCoNi NLTE model $\left(T_{\text {eff }}=42 \mathrm{kK}\right.$, log $\left.g=5.3\right)$ (blue, thin line, Kurucz' POS lines) and the synthetic stellar + ISM spectrum (thick red, Kurucz LIN lines) with the FUSE observation. The observation is shifted to match the rest wavelengths of the photospheric lines marked at the top of the panels. The synthetic spectra are normalized to match the observed flux level with a factor linearly increasing from $5.6 \times 10^{18}$ to $7.9 \times 10^{18}$ within $910 \AA-1190 \AA$. 
Table 2. Identified photospheric lines in the FUSE observation of LB 3459, with only P IV $3 p{ }^{3} \mathrm{P}^{\circ}-3 \mathrm{p}^{2}{ }^{3} \mathrm{P}$ lines identified, and other P IV line identifications uncertain.

\begin{tabular}{|c|c|c|}
\hline Wavelength / Å & Ion & Transition \\
\hline 918.50 & P IV & $3 p^{2}{ }^{1} D-3 d^{1} D^{o}$ \\
\hline 920.55 & He II & $2-20$ \\
\hline 920.96 & $\mathrm{H} \mathrm{I}$ & $1-10$ \\
\hline 921.32 & O IV & $2 \mathrm{p}^{2}{ }^{2} \mathrm{P}-2 \mathrm{p}^{3}{ }^{2} \mathrm{P}^{o}$ \\
\hline 921.56 & He II & $2-19$ \\
\hline $921.99-924.28$ & N IV & $2 \mathrm{p}^{3} \mathrm{P}^{\mathrm{o}}-2 \mathrm{p}^{2}{ }^{3} \mathrm{P}$ \\
\hline 922.74 & He II & $2-18$ \\
\hline 923.15 & $\mathrm{H} \mathrm{I}$ & $1-9$ \\
\hline 924.13 & He II & $2-17$ \\
\hline 924.22 & S V & $3 p^{1} \mathrm{P}^{\mathrm{o}}-3 \mathrm{p}^{2}{ }^{1} \mathrm{~S}$ \\
\hline 925.81 & He II & $2-16$ \\
\hline 926.23 & $\mathrm{H} \mathrm{I}$ & $1-8$ \\
\hline 927.85 & He II & $2-15$ \\
\hline 930.34 & He II & $2-14$ \\
\hline 930.75 & $\mathrm{H} \mathrm{I}$ & $1-7$ \\
\hline $933.38-944.52$ & S VI & $3 s^{2} S-3 p{ }^{2} P^{o}$ \\
\hline 933.45 & $\mathrm{He}$ II & $2-13$ \\
\hline 937.39 & He II & $2-12$ \\
\hline 937.80 & $\mathrm{H} \mathrm{I}$ & $1-6$ \\
\hline 942.51 & He II & $2-11$ \\
\hline $948.09-948.21$ & C IV & $3 s^{2} S-4 p{ }^{2} P^{o}$ \\
\hline $948.15-948.61$ & N IV & $3 p^{3} \mathrm{P}^{\mathrm{o}}-4 \mathrm{~d}{ }^{3} \mathrm{D}$ \\
\hline 949.33 & He II & $2-10$ \\
\hline 949.74 & $\mathrm{H} \mathrm{I}$ & $1-5$ \\
\hline 950.66 & P IV & $3 \mathrm{~s}^{2}{ }^{1} \mathrm{~S}-3 \mathrm{p}{ }^{1} \mathrm{P}^{\mathrm{o}}$ \\
\hline 955.34 & $\mathrm{~N}$ IV & $2 \mathrm{p}^{1} \mathrm{P}^{\mathrm{o}}-2 \mathrm{p}^{2}{ }^{1} \mathrm{~S}$ \\
\hline 958.70 & He II & $2-9$ \\
\hline $967.66-968.18$ & $\mathrm{O}_{\mathrm{III}}$ & $2 p^{31} P^{o}-3 p{ }^{1} S$ \\
\hline 972.11 & He II & $2-8$ \\
\hline $\begin{array}{l}972.53 \\
975.84\end{array}$ & $\begin{array}{l}\mathrm{H} \mathrm{I} \\
\mathrm{S} \text { VI }\end{array}$ & $4 p^{2} \mathrm{P}^{\mathrm{o}}-5 \mathrm{~s}^{2} \mathrm{~S}$ \\
\hline 977.02 & C III & $2 \mathrm{~s}^{2}{ }^{1} \mathrm{~S}-2 \mathrm{p}{ }^{1} \mathrm{P}^{\mathrm{o}}$ \\
\hline $979.77-979.97$ & N III & $2 \mathrm{p}^{2} \mathrm{D}-2 \mathrm{p}^{3} \mathrm{D}^{0}$ \\
\hline $989.80-991.58$ & N III & $2 p^{2} P^{o}-2 p^{2}{ }^{2} D$ \\
\hline 992.36 & He II & $2-7$ \\
\hline $997.54-1000.38$ & $\mathrm{P} V$ & $3 d^{2} D-4 p^{2} P^{o}$ \\
\hline $1000.37-1000.54$ & S VI & $4 d^{2} D-5 f^{2} F^{o}$ \\
\hline $1001.75-1003.21$ & N III & $2 p^{2}{ }^{2} \mathrm{P}-3 \mathrm{p}^{2} \mathrm{P}^{\mathrm{o}}$ \\
\hline $1005.99-1006.03$ & N III & $2 \mathrm{p}^{2}{ }^{2} \mathrm{~S}-2 \mathrm{p}^{3} \mathrm{p}^{\mathrm{o}}$ \\
\hline 1006.23 & $\mathrm{P}$ IV & $3 p^{2}{ }^{1} D-4 p{ }^{1} \mathrm{P}^{o}$ \\
\hline 1025.27 & He II & $2-6$ \\
\hline 1025.56 & P IV & $3 p^{3} \mathrm{P}^{0}-3 p^{23} \mathrm{P}$ \\
\hline 1025.72 & $\mathrm{H} \mathrm{I}$ & $1-3$ \\
\hline $1028.09-1035.52$ & P IV & $3 p^{3} \mathrm{P}^{\mathrm{o}}-3 \mathrm{p}^{2}{ }^{3} \mathrm{P}$ \\
\hline 1039.92 & $\mathrm{~S} \mathrm{~V}$ & $3 d^{1} D-3 d^{1} F^{o}$ \\
\hline $1045.50-1047.27$ & Si IV & $4 p^{2} P^{0}-6 d^{2} D$ \\
\hline 1049.65 & P IV & $3 d^{1} D-3 d^{1} F^{o}$ \\
\hline 1050.60 & N IV & $3 p^{1} \mathrm{P}^{0}-3 \mathrm{p}^{1} \mathrm{D}$ \\
\hline 1062.88 & S IV & $3 p^{2} \mathrm{P}^{0}-3 p^{2} \mathrm{D}$ \\
\hline $1064.61-1066.64$ & P IV & $3 d^{3} D-3 d^{1} F^{o}$ \\
\hline $1073.19-1073.72$ & S IV & $3 p^{2} \mathrm{P}^{o}-3 p^{2} \mathrm{D}$ \\
\hline 1078.71 & N IV & $3 \mathrm{~d}^{1} \mathrm{D}-4 \mathrm{f}{ }^{1} \mathrm{~F}^{\mathrm{o}}$ \\
\hline 1084.94 & He II & $2-5$ \\
\hline $1086.08-1086.69$ & $\mathrm{~N}$ IV & $3 p^{3} \mathrm{P}^{\mathrm{o}}-3 \mathrm{p}^{3} \mathrm{~S}$ \\
\hline $1086.93-1091.44$ & P IV & $3 d^{3} D-3 d^{3} D^{o}$ \\
\hline $1092.35-1093.48$ & N IV & $3 d^{3} D-3 d^{3} P^{o}$ \\
\hline $1097.32-1097.34$ & $\mathrm{C}$ IV & $4 s^{2} S-8 p^{2} \mathrm{P}^{o}$ \\
\hline 1104.53 & N IV & $3 d^{3} F^{0}-4 p^{3} D$ \\
\hline $1107.59-1107.98$ & $\mathrm{C}$ IV & $3 p^{2} \mathrm{P}^{0}-4 \mathrm{~d}^{2} \mathrm{D}$ \\
\hline 1117.76 & S VI & $4 \mathrm{f}^{2} \mathrm{~F}^{0}-5 \mathrm{~g}^{2} \mathrm{G}$ \\
\hline 1117.93 & N IV & $3 d^{1} D-3 d^{1} P^{o}$ \\
\hline $1117.98-1128.01$ & $\mathrm{P} V$ & $3 s^{2} S-3 p^{2} \mathrm{P}^{o}$ \\
\hline 1118.55 & P IV & $3 p^{1} \mathrm{P}^{0}-3 \mathrm{p}^{21} \mathrm{~s}$ \\
\hline $1122.03-1134.09$ & $\mathrm{~S} \mathrm{~V}$ & $3 d^{3} D-3 d^{3} F^{o}$ \\
\hline $1122.49-1128.34$ & Si IV & $3 p^{2} P^{o}-3 d^{2} D$ \\
\hline $1131.03-1132.94$ & N IV & $3 p^{3} \mathrm{P}^{0}-3 p^{3} \mathrm{P}$ \\
\hline $1133.12-1136.27$ & N IV & $3 s^{3} \mathrm{~S}-3 \mathrm{~s}{ }^{3} \mathrm{P}^{0}$ \\
\hline 1137.28 & P IV & $3 d^{1} D-3 d^{1} P^{o}$ \\
\hline $1149.63-1153.78$ & $\mathrm{O}$ III & $2 p^{3}{ }^{3} s^{o}-2 p^{43} p$ \\
\hline $1168.48-1169.57$ & N IV & $3 d^{3} D-3 d^{3} D^{o}$ \\
\hline $1168.86-1169.01$ & $\mathrm{C}$ IV & $3 d^{2} D-4 f^{2} F^{o}$ \\
\hline $1174.93-1176.37$ & $\mathrm{C}$ III & $2 p^{3} P^{0}-2 p^{2}{ }^{3}$ \\
\hline $1182.97-1184.57$ & N III & $2 \mathrm{p}^{2} \mathrm{P}-2 \mathrm{p}^{3} \mathrm{P}^{\mathrm{o}}$ \\
\hline
\end{tabular}

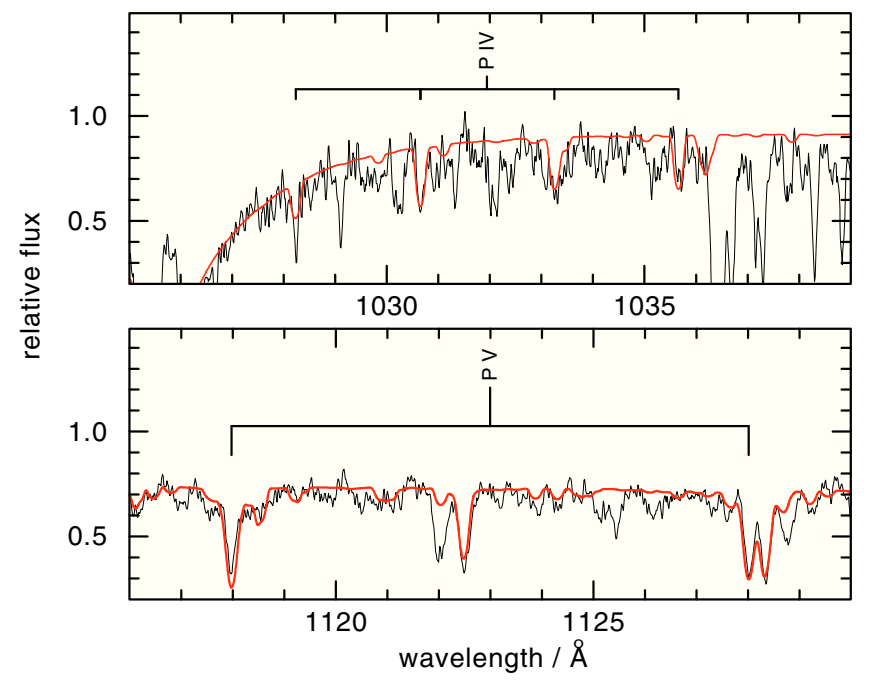

Fig. 8. Comparison of synthetic spectra ( $T_{\mathrm{eff}}=42 \mathrm{kK}, \log g=5.3$, P with solar abundance) around P IV $\lambda \lambda 1028.09,1030.51,1033.11,1035.51 \AA$ (top) and the $\mathrm{P} \vee \lambda \lambda 1117.98,1128.01 \AA$ resonance doublet (bottom) with the FUSE observation.

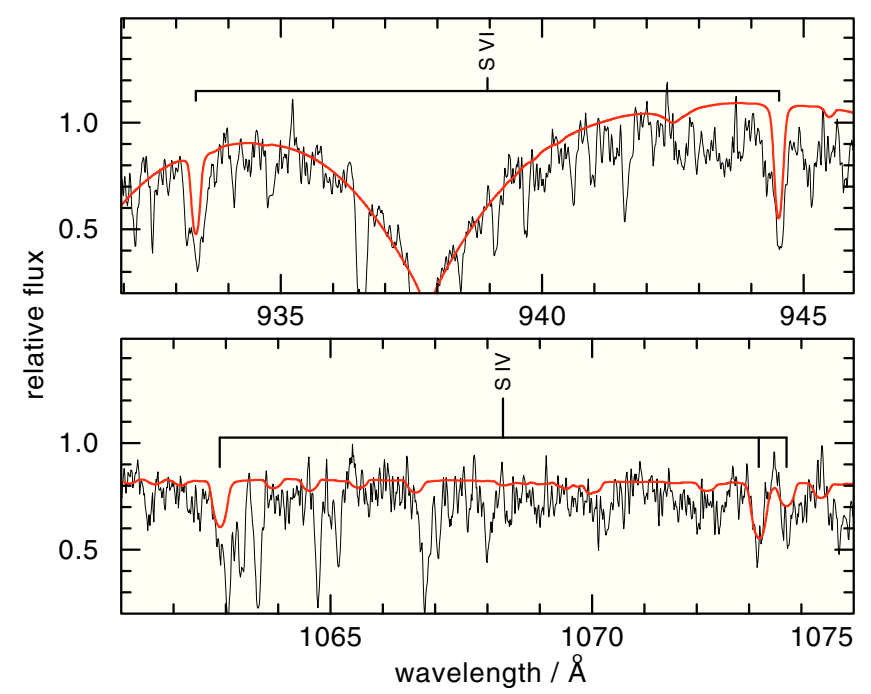

Fig. 9. Comparison of synthetic spectra $\left(T_{\text {eff }}=42 \mathrm{kK}, \log g=5.3\right.$, sulfur with 0.01 times solar abundance) around the $\mathrm{S}$ VI $\lambda \lambda 933.38,944.52 \AA$ resonance doublet (top) and S IV $\lambda \lambda 1062.88,1073.19,1073.72 \AA$ (bottom) with the FUSE observation.

\section{Spectral analysis}

A detailed spectral analysis of optical and near-UV observations LB 3459 had been performed by Rauch (2000). We considered additional species (Table 1) in order to model the far-UV spectrum reliably. Although no iron-group lines were identified in the FUSE observation, we included all iron-group elements with individual model atoms (Table 1) using the Kurucz line lists (Kurucz 1991). The model atoms and the respective atomic data files were constructed in a statistical approach (introducing "superlevels" and "superlines") with the program IrOnIc (Iron Opacity Interface, Deetjen et al. 1999; Rauch \& Deetjen 2003). For the model-atmosphere calculation, we considered all lines (so-called LIN lists that include laboratory measured, as well as theoretically calculated lines) to simulate the total opacity correctly. Figure 7 shows the good agreement of our final synthetic "LIN" spectrum with the FUSE observation. In a close inspection, e.g. around $\lambda 960 \AA$ or $\lambda 1015 \AA$, opacity appears to be 


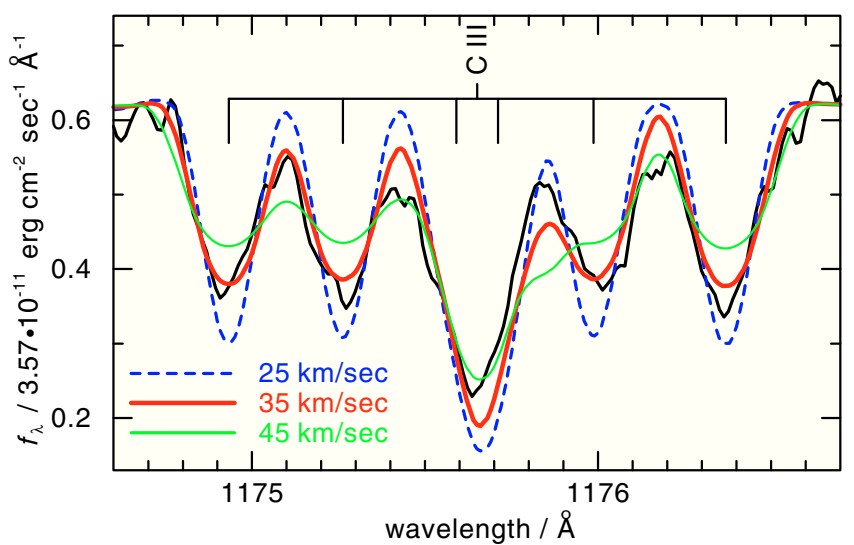

Fig. 10. Comparison of our synthetic spectrum $\left(T_{\text {eff }}=42 \mathrm{kK}, \log g=5.3\right)$ with the FUSE observation around $C_{\text {III }} \lambda \lambda 1174.93-1176.37 \AA$. The synthetic spectrum is convolved with rotational profiles $\left(v_{\text {rot }}=\right.$ $25,35,45 \mathrm{~km} \mathrm{~s}^{-1}$ ). The best fit is achieved at $v_{\text {rot }}=35 \mathrm{~km} \mathrm{~s}^{-1}$.

missing. This might be the result of partly uncertain "LIN"-line wavelengths.

For a detailed comparison with the observations, e.g. for identification and abundance determination, we have to restrict ourselves to lines measured in the laboratory (using POS lists about $10 \%$ of the lines in the LIN lists; Fig. 7 ).

\subsection{Rotational velocity}

The spectral analysis of Rauch (2000) was hampered by an uncertain rotational velocity. The strongest photospheric line feature in the FUSE observation of LB 3459 (Fig. 7), C III $\lambda \lambda 1174-1177 \AA$, is well-suited to measuring the rotational velocity (Fig. 10). We determined $v_{\text {rot }}=35 \pm 5 \mathrm{~km} \mathrm{~s}^{-1}$. This agrees with $v_{\text {rot }}=34 \pm 10 \mathrm{~km} \mathrm{~s}^{-1}$, which was calculated by Rauch (2000) in an attempt to numerically eliminate the effects of orbital smearing from their spectra with relatively long exposure times. However, Rauch (2000) and Rauch \& Werner (2003) assumed this $v_{\text {rot }}$ was uncertain and used $v_{\text {rot }}=45 \mathrm{~km} \mathrm{~s}^{-1}$ in their analyses. Our result now agrees with the $v_{\text {rot }}=34.7-38.7 \mathrm{~km} \mathrm{~s}^{-1}$ calculated under the assumption of bound rotation from the primary's radius $R_{1}=0.179-0.200 R_{\odot}$ given by Hilditch et al. (2003).

From $v_{\text {rot }}=35 \pm 5 \mathrm{~km} \mathrm{~s}^{-1}$ and an orbital period of $P=$ $22597.033 \mathrm{~s}$ (Kilkenny et al. 2000), we can calculate a stellar radius of $r_{1}=0.181 \pm 0.025 R_{\odot}$. Rauch \& Werner (2003) measured a radial-velocity amplitude of $A_{1}=39.19 \pm 0.05 \mathrm{~km} \mathrm{~s}^{-1}$ and calculated $a_{1}=0.2025 \pm 0.0019 R_{\odot}$. With $a_{1}=0.2025 R_{\odot}$ and $r_{1}=0.181 R_{\odot}$, we can calculate $v_{\text {rot }}=31 \mathrm{~km} \mathrm{~s}^{-1}$, which is in good agreement. Thus, it is most likely that the rotation of LB 3459 is bound.

\subsection{Effective temperature}

The effective temperature $T_{\text {eff }}=42 \pm 1 \mathrm{kK}$ of LB 3459 was determined by Rauch (2000) within small error limits from the evaluation of ionization equilibria of $\mathrm{He}$ i/He II, C III/C IV, N III-N v, and $\mathrm{O}$ IV/O v. In the FUSE wavelength range, we also find metal lines of successive ionization stages, namely $\mathrm{C}$ III/C IV, $\mathrm{N}$ III $/ \mathrm{N}$ IV, $\mathrm{O}$ III/O IV, $\mathrm{P}$ IV $/ \mathrm{P}$ v, and $\mathrm{S}$ IV $/ \mathrm{S}$ v. Due to the strong contamination by the ISM, many of these lines, e.g. the strong C III $\lambda 977.03 \AA$ and $\mathrm{N}$ III $\lambda$ 989.79 $\AA$ (Fig. 7) lines, cannot be used, but there are isolated lines that are suitable for a analogous determination

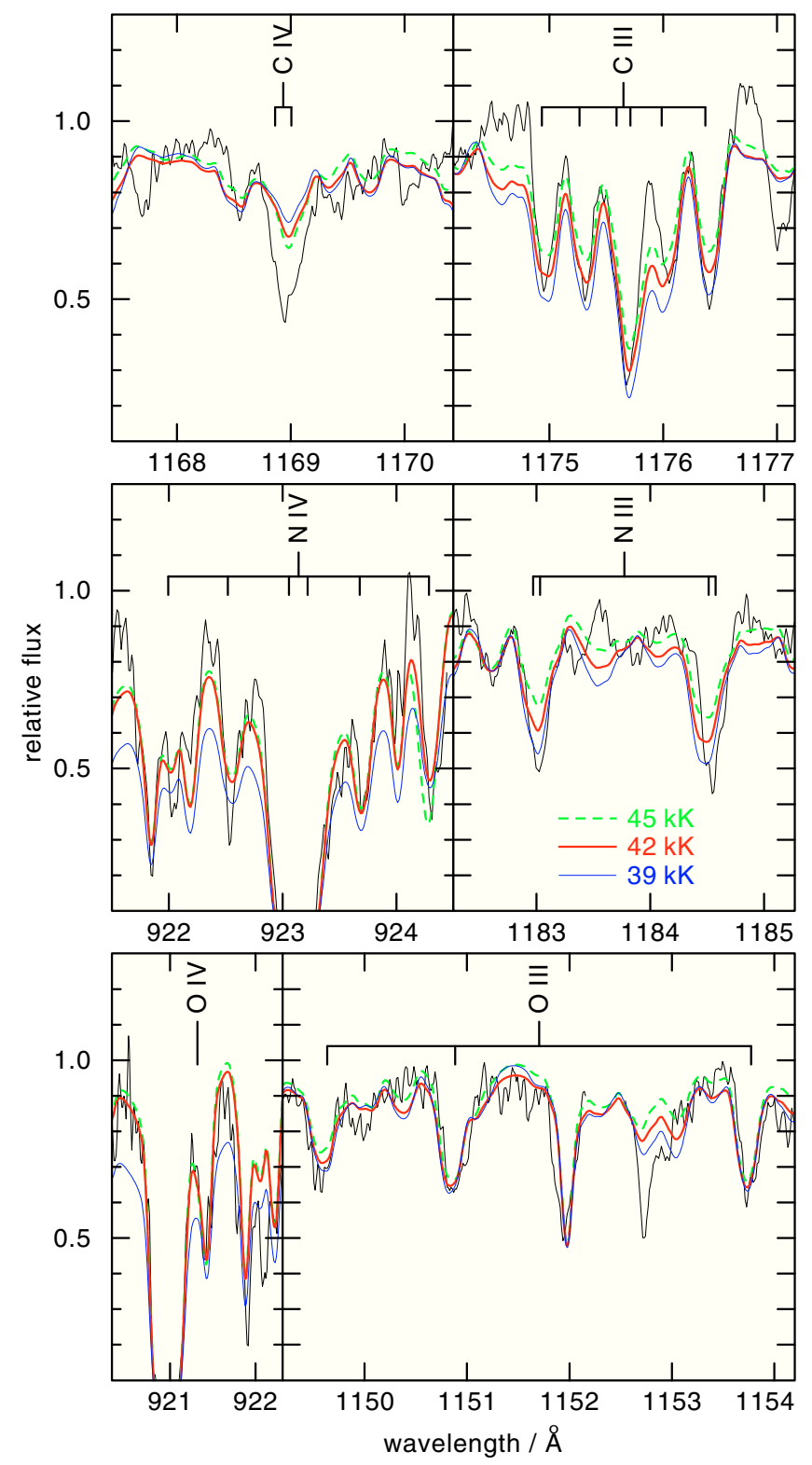

Fig. 11. Comparison of synthetic spectra $(\log g=5.3)$ around selected $\mathrm{C}$ IV and C III (top panel), N IV and N III (middle), O IV and O III (bottom) lines of models calculated with $T_{\text {eff }}=39,42,45 \mathrm{kK}$ with the FUSE observation. We use models calculated with Kurucz' LIN lines in order to demonstrate the agreement with the local continuum.

of $T_{\text {eff }}$ (Figs. 8, 9, 11). Their ionization equilibria appear wellmatched at $T_{\text {eff }}=42 \mathrm{kK}$, however, the strong contamination by ISM line absorption, the reddening, and the iron-group opacities (Sect. 4.4) make it difficult to find the continuum flux level for a proper normalization. Thus, we estimate that our $T_{\text {eff }}$ determination cannot be better than $\pm 3 \mathrm{kK}$. Consequently, we adopted $T_{\text {eff }}=42 \mathrm{kK}$ for our analysis.

\subsection{Surface gravity}

To investigate the impact of the rotational velocity on the determination of the surface gravity of LB 3459, we repeated the $\chi^{2}$ fit of Rauch (2000, see his Fig. 4) with the same synthetic and observed fluxes, but we used $v_{\text {rot }}=35 \pm \mathrm{km} \mathrm{s}^{-1}$. We arrive now at a higher $\log g=5.30(\Delta \log g=0.09)$. These synthetic fluxes were calculated only from $\mathrm{H}+\mathrm{He}$ models, so 


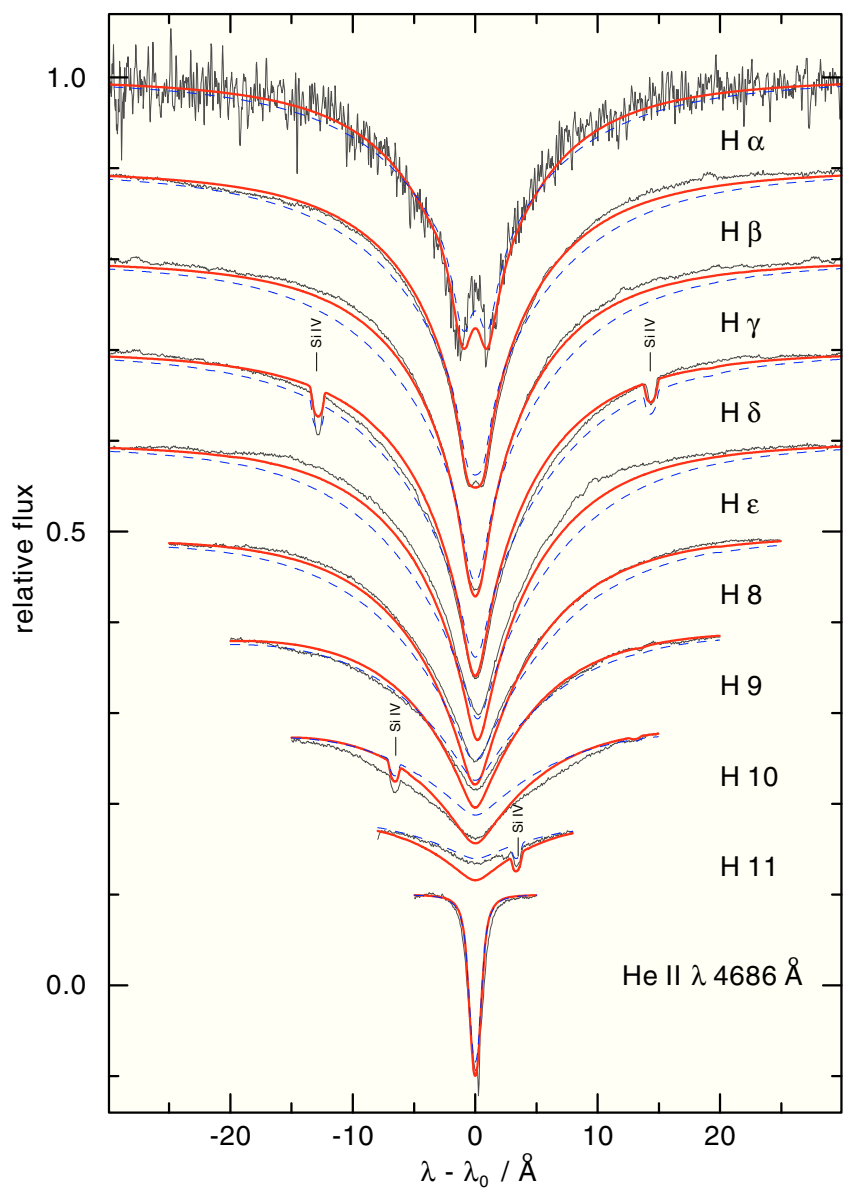

Fig. 12. Synthetic Balmer-line profiles calculated with $T_{\text {eff }}=42 \mathrm{kK}$ and $\log g=5.3$ (thick red line) and $\log g=5.6$ (dashed, blue) compared with with our co-added (all 105 exposures) UVES observation. A surface gravity as high as $\log g=5.6$ can definitely be excluded. The normalization of the observation to the continuum is not perfect for the broad Balmer lines. For the narrow He II $\lambda 4686 \AA$ line, the $\log g$ dependence is visible only in the innermost core.

they suffer from the Balmer-line problem due to the neglecting metal opacities (Werner 1996). Consequently, we calculated a small grid of models that consider all elements from $\mathrm{H}-\mathrm{Ni}$. Due to the relatively long calculation times of these much more detailed models, we had to restrict this grid to a fixed $T_{\text {eff }}=42 \mathrm{kK}, \log g=5.20-5.60$ and $\Delta \log g=0.05$. The $\chi^{2}$ fit yields $\log g=5.30$ for $\mathrm{H} \alpha$ and $\mathrm{H} \epsilon$ and $\log g=5.35$ for $\mathrm{H} \beta, \mathrm{H} \gamma$, and $\mathrm{H} \delta$. A comparison of the observed hydrogen Balmer series with the observations (Fig. 12) shows clearly that a higher $\log g$ does not agree with the observations.

In the FUSE wavelength region, a higher surface gravity seems to improve the fit to the "shoulders" between the higher hydrogen Lyman lines (Fig. 13). However, the many uncertainties in the wavelengths range, e.g. due to ISM absorption, unfortunately prevent any firm conclusion.

\subsection{Abundances}

The possibility of determining photospheric abundances from the FUSE observation is strongly limited by both the ISM line absorption (Fig. 7) and the limited number of Kurucz' POS lines (Sect.4). Only a few isolated photospheric lines are accessible in this wavelength range. We adopted the previously determined abundances given by Rauch (2000) and the adjusted Si and Ni

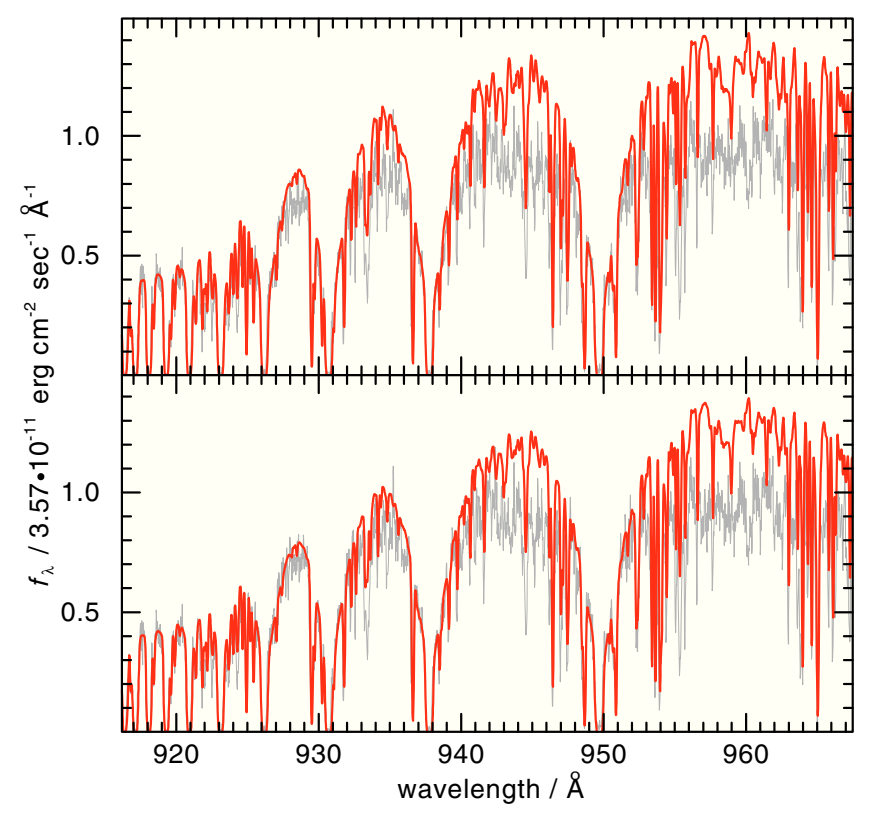

Fig. 13. Synthetic spectra calculated with $T_{\text {eff }}=42 \mathrm{kK}$ and $\log g=5.3$ (top panel) and $\log g=5.6$ (bottom) compared with with our FUSE observation of LB 3459.

abundances (Sect. 3), because test calculations have shown that these give a good fit to the observation. The newly introduced elements phosphorus and sulfur enabled us to identify lines of P IV and $\mathrm{P}$ V (Fig. 8) and S IV and S VI (Fig. 9). The determined abundances are solar and 0.01 times solar, respectively. Only weak lines of the iron-group element are located in the FUSE wavelength range. Due to the stellar rotation (Sect.4.1), we were not able to identify any of these lines.

\section{Results and conclusions}

We performed an NLTE spectral analysis of FUSE observations of the post common-envelope binary LB 3459. The short FUSE exposure times $(200 \mathrm{~s})$ allowed us to measure the rotational velocity $v_{\text {rot }}=35 \pm 5 \mathrm{~km} \mathrm{~s}^{-1}$ of the primary star of LB 3459 . This is in good agreement with the $v_{\mathrm{rot}}=34.7-38.6 \mathrm{~km} \mathrm{~s}^{-1}$ given by Hilditch et al. (2003), and it confirms that the rotation of LB 3459 is bound.

A re-analysis of optical spectra (cf. Rauch 2000) has shown that $\log g$ is about 0.1 higher than given by Rauch (2000, $\log g=5.2$ ), who assumed bound rotation because the system has been classified to be a post common-envelope binary consisting of an SdOB and a main-sequence star (de Kool \& Ritter 1993) where the common-envelope phase is much longer than the synchronization time. Since the primary's radius was assumed to be larger $\left(r_{1}=0.236 R_{\odot}\right)$ due to the lower $\log g$, a higher $v_{\text {rot }}=45 \mathrm{~km} \mathrm{~s}^{-1}$ was adopted then for the analysis. From $v_{\text {rot }}=35 \pm 5 \mathrm{~km} \mathrm{~s}^{-1}$ and an orbital period of $P=22597.033 \mathrm{~s}$ (Kilkenny et al. 2000), we can calculate a stellar radius of $r_{1}=0.181 \pm 0.025 R_{\odot}$.

The spectral analysis is hampered by a strong ISM contamination. We used OWENS to model the ISM line absorption qualitatively in order to identify and model stellar lines. Our NLTE models consider opacities of 18 species from $\mathrm{H}-\mathrm{Ni}$. It is obvious that the iron-group elements (here: $\mathrm{Ca}-\mathrm{Ni}$ ) contribute to the opacity. However, we were not able to identify any individual iron-group line in the FUSE observation. 


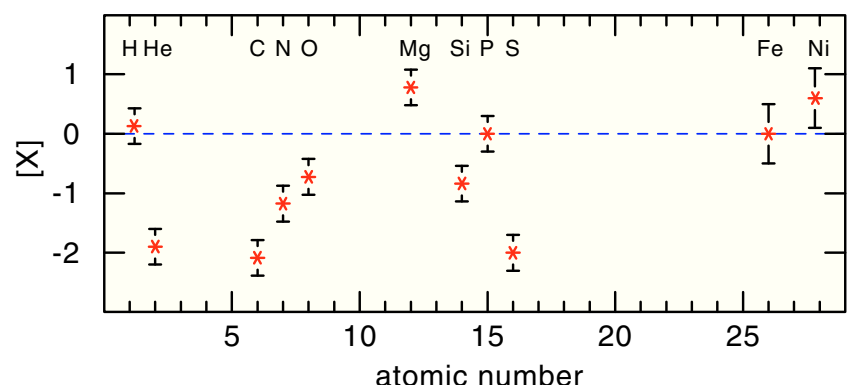

Fig. 14. Photospheric abundances of LB 3459 determined by Rauch (2000) and in this work ( $\mathrm{Si}, \mathrm{P}, \mathrm{S}, \mathrm{Ni})$. [X] denotes $\log$ (mass fraction/solar mass fraction) of species X.

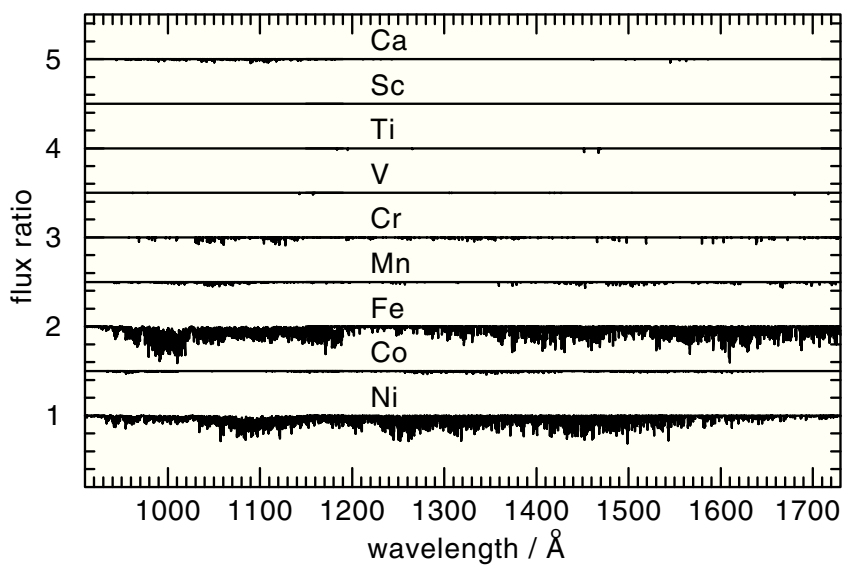

Fig. 15. Flux ratios (in the far UV) of synthetic spectra for LB 3459 (calculated with abundances from Fig. 14 and Kurucz's LIN lines), which include only line opacities of one of the elements $\mathrm{Ca}-\mathrm{Ni}$ and a synthetic spectrum, which does not include any $\mathrm{Ca}-\mathrm{Ni}$ line.

We identified phosphorus and sulfur lines in the FUSE spectrum. For phosphorus we can determine a solar abundance. The sulfur abundance is surprisingly low, and we determined a 0.01 times solar abundance. The photospheric abundances of LB 3459 are summarized in Fig. 14. The derived abundance pattern reflects the interplay of gravitational settling and radiative levitation (cf. Rauch 2000).

The "gravity problem" is still unsolved although the surface gravity is slightly higher: $\log g=5.3$ rather than the 5.2 found by Rauch (2000). If we take the radius of the primary as $r_{1}=$ $0.181 R_{\odot}$ as noted above, and its mass $M_{1}=0.330 M_{\odot}$ (Rauch 2000), then we obtain $\log g=\log \left(G M_{1} / R_{1}^{2}\right)=5.44 \pm 0.13$ (where $G$ is the gravitational constant). Note that $\log g \approx 5.5$ is necessary for good agreement (cf. Rauch 2000).

An additional uncertainty (cf. Rauch 2000) is the lack of appropriate evolutionary models for post common-envelope binaries to compare so as to derive the primary's mass.

High-resolution and high $\mathrm{S} / \mathrm{N}$ observations in the near-UV wavelength range is highly desirable when searching for the weak lines of iron-group elements. Figure 15 demonstrates that lines of all elements from $\mathrm{Ca}-\mathrm{Ni}$ but $\mathrm{Sc}$ and V should be detectable. The search for signatures of the "heated-up" secondary ( $T_{\text {eff }}$ increased to about $16 \mathrm{kK}$ by irradiation of the primary) to further investigate the nature of this star also appears possible, and its contribution to the continuum at $\lambda=12 \mu$ is a few percent.

Acknowledgements. We like to thank our referee, Dave Kilkenny, for his useful comments that helped us to clarify and improve this paper. T.R. is supported by the German Astrophysical Virtual Observatory (GAVO) project of the German Federal Ministry of Education and Research (BMBF) under grant 05 AC6VTB. J.W.K. is supported by the FUSE project, funded by NASA contract NAS5-32985. This work was done using the profile-fitting procedure OWENS developed by M. Lemoine and the FUSE French Team. This work is based on INES ${ }^{12}$ data from the IUE satellite. Some of the data presented in this paper were obtained from the Multimission Archive at the Space Telescope Science Institute (MAST). STScI is operated by the Association of Universities for Research in Astronomy, Inc., under NASA contract NAS5-26555. Support for MAST for non-HST data is provided by the NASA Office of Space Science via grant NAG5-7584 and by other grants and contracts. The UVES spectra used in this analysis were obtained as part of an ESO Service Mode run, proposal 66.D-1800. This research has made use of the SIMBAD database, operated at the CDS, Strasbourg, France.

\section{References}

Asplund, M., Grevesse, N., \& Sauval, A. J. 2005, in Cosmic Abundances Records of Stellar Evolution and Nucleosynthesis, ed. T. G. Barnes III., \& F. N. Bash (San Francisco: ASP), ASP Conf. Ser., 336, 25

Deetjen, J. L., Dreizler, S., Rauch, T., \& Werner, K. 1999, A\&A, 348, 940 de Kool, M., \& Ritter, H. 1993, A\&A, 267, 397

Dixon, W. V., Sahnow, D. J., Barrett, P. E., et al. 2007, PASP, 119, 527

Driebe, T., Schönberner, D., Blöcker, T., \& Herwig, F. 1998, A\&A, 339, 123

Groenewegen, M. A. T., \& Lamers, H. J. G. L. M. 1989, A\&AS, 79, 359 Hébrard, G., Lemoine, M., Vidal-Madjar, A., et al. 2002, ApJS, 140, 103 Hilditch, R. W., Harries, T. J., \& Hill, G. 1996, MNRAS, 279, 1380

Hilditch, R. W., Kilkenny, D., Lynas-Gray, A. E., \& Hill, G. 2003, MNRAS, 344, 644

Howarth, I. 1983, MNRAS, 203, 301

Hummer, D. G., Berrington, K. A., Eissner, W., et al. 1993, A\&A, 279, 298

Kilkenny, D., Lynas-Gray, A. E., \& Hilditch, R. W. 1979, White Dwarfs and Variable Degenerate Stars, IAU Colloq., 53, 255

Kilkenny, D., Hill, P. W., \& Penfold, J. E. 1981, MNRAS, 194, 429

Kilkenny, D., Keuris, S., Marang, F., et al. 2000, The Observatory, 120, 48

Kruk, J. W., Kimble, R. A., Buss, R. H. Jr., et al. 1997, ApJ, 482, 546

Kruk, J. W., Howk, J. C., André, M., et al. 2002, ApJS, 140, 19

Kudritzki, R. P., Simon, K. P., Lynas-Gray, A. E., Kilkenny, D., \& Hill, P. W. 1982, A\&A, 106, 254

Kurucz, R. L. 1991, in Stellar Atmospheres: Beyond Classical Models, ed. L. Crivellari, I. Hubeny, \& D. G. Hummer, NATO ASI Series C (Dordrecht: Kluwer), 341, 441

Lemoine, M., Vidal-Madjar, A., Hébrard, G., et al. 2002, ApJS, 140, 67

Lynas-Gray, A. E., Heber, U., Kudritzki, R. P., \& Simon, K. P. 1984, in Fourth European IUE Conference, ESA SP-218, 285

Moos, H. W., Cash, W. C., Cowie, L. L., et al. 2000, ApJ, 538, L1

Paczynski, B. 1980, Acta Astron., 30, 113

Rauch, T. 2000, A\&A, 356, 665

Rauch, T. 2004, Ap\&SS, 291, 275

Rauch, T., \& Deetjen, J. L. 2003, in Stellar Atmosphere Modeling, ed. I. Hubeny, D. Mihalas, \& K. Werner (San Francisco: ASP), ASP Conf. Ser., 288, 103

Rauch, T., \& Werner, K. 2003, A\&A, 400, 271

Sahnow, D. J., Moos, H. W., Ake, T. B., et al. 2000, ApJ, 538, L7

Savitzky, A., \& Golay M. J. E. 1964, Analytical Chemistry, 36, 1627

Seaton, M. J. 1979, MNRAS, 187, 73

Seaton, M. J., Yan, Y., Mihalas, D., \& Pradhan, A. K. 1994, MNRAS, 266, 805

Werner, K., Deetjen, J. L., Dreizler, et al. 2003, in Stellar Atmosphere Modeling, eds. I. Hubeny, D. Mihalas, \& K. Werner (San Francisco: ASP), ASP Conf. Ser., 288, 31

Werner, K. 1996, ApJ, 457, L39 